\title{
Long-Term Outcome of Low-Energy Extracorporeal Shock Wave Therapy for Plantar Fasciitis: Comparative Analysis According to Ultrasonographic Findings
}

\author{
Jong-Wan Park, $\mathrm{MD}^{1}$, Kyungjae Yoon, $\mathrm{MD}, \mathrm{PhD}^{1}$, Kwang-Soo Chun, $\mathrm{MD}^{1}$, \\ Joon-Youn Lee, $\mathrm{MD}^{1}$, Hee-Jin Park, $\mathrm{MD}^{2}$, So-Yeon Lee, $\mathrm{MD}^{2}$, Yong-Taek Lee, MD, PhD ${ }^{1}$ \\ Departments of ${ }^{1}$ Physical and Rehabilitation Medicine, ${ }^{2}$ Radiology, Kangbuk Samsung Hospital, \\ Sungkyunkwan University School of Medicine, Seoul, Korea
}

Objective To investigate the long-term effect of low-energy extracorporeal shock wave therapy (ESWT) for plantar fasciitis (PF) according to ultrasonography (US) findings.

Methods Thirty feet of 25 patients with clinical diagnosis of PF were enrolled and divided into two groups (Apparent-US and Uncertain-US) according to US findings, such as plantar fascia thickening or hypoechogenicity. Inclusion criteria were symptom duration $>6$ months and a fair or poor grade in Roles-Maudsley score (RMS). ESWT $\left(0.10 \mathrm{~mJ} / \mathrm{mm}^{2}, 600\right.$ shocks) was given once a week for 6 weeks. Numeric rating scale (NRS) and RMS were evaluated prior to each ESWT session, at short-term follow-up (one week after all ESWT sessions) and long-term follow-up telephone interview (mean 24 months after ESWT). Good and excellent grade in RMS were considered as treatment success.

Results Repeated measure ANOVA demonstrated that NRS significantly decreased with time after ESWT up to the long-term follow-up (time effect, $\mathrm{p}<0.001)$ without group-time interaction $(\mathrm{p}=0.641)$, indicating that ESWT equally decreased pain in both groups. Overall success rate was $63.3 \%$ (short-term follow-up) and $80.0 \%$ (long-term follow-up). In comparative analysis between groups, success rate of Apparent-US and Uncertain-US at short-term follow-up was $61.9 \%$ and $66.7 \%$, respectively, and $85.7 \%$ and $66.7 \%$, respectively, at long-term follow-up.

Conclusion If other causes of heel pain are ruled out through meticulous physical examination and ultrasonography, low-energy ESWT in PF seems to be beneficial regardless of US findings. In terms of success rate, however, long-term outcome of Apparent-US appears to be superior to Uncertain-US.

Keywords Plantar fasciitis, Extracorporeal shock wave therapy (ESWT), Ultrasonography, Treatment outcome licenses/by-nc/3.0) which permits unrestricted noncommercial use, distribution, and reproduction in any medium, provided the original work is properly cited. Copyright $\odot 2014$ by Korean Academy of Rehabilitation Medicine 


\section{INTRODUCTION}

Plantar fasciitis (PF) is the most common cause of heel pain and accounts for $11 \%$ to $15 \%$ of all foot symptoms requiring medical care [1-3]. The standard treatments of PF are conservative measures including insoles, shoe modification, physical therapy, stretching exercises and nonsteroidal anti-inflammatory drugs (NSAIDs). Further approaches include corticosteroid injections [4-8]. For intractable cases, surgical release of the plantar fascia may be considered. Extracorporeal shock wave therapy (ESWT) has been increasingly used as a safe alternative treatment option for these, since many published papers have reported a beneficial effect [9-15].

The diagnosis of PF has been typically based on clinical history and the finding of localized tenderness [14]. Recently, ultrasonography (US) has received increased attention for its diagnostic capabilities, since many studies have reported the thickening and hypoechoic changes of the plantar fascia as characteristic features of PF [16-19]. Thus, nowadays diagnosis of PF is often made based on both clinical and US findings. However, only clinical findings have been used for the diagnosis of PF in the majority of previous outcome studies for ESWT. Based on these studies, the United States Food and Drug Administration approved ESWT for the treatment of clinically diagnosed chronic intractable PF.

To our knowledge, no study has addressed the outcome of ESWT involving patients with clinically diagnosed PF but whose US abnormality was uncertain. The present study was undertaken to investigate the therapeutic effect of low-energy ESWT in these patients and to compare the results with clinically diagnosed and US confirmed PF.

\section{MATERIALS AND METHODS}

\section{Subjects}

Between 2008 and 2013, 70 patients (96 feet) with

Table 1. Roles-Maudsley score

\begin{tabular}{lcl}
\hline & Point & \multicolumn{1}{c}{ Interpretation } \\
\hline Excellent & 1 & No pain, full movement and activity \\
Good & 2 & $\begin{array}{l}\text { Occasional discomfort, full movement } \\
\text { and activity }\end{array}$ \\
Fair & 3 & Some discomfort after prolonged activity \\
Poor & 4 & Pain-limiting activities \\
\hline
\end{tabular}

chronic refractory PF were enrolled. Diagnosis of PF was made based on the clinical findings of the patients' history and physical examination. As an additional tool, US evaluation was performed to confirm the $\mathrm{PF}$ and rule out other diseases. Among them, 25 patients (30 feet) met the inclusion and exclusion criteria. Inclusion criteria were as follows: PF of at least 6 months in duration, lack of pain relief by standard treatments including NSAIDs, insoles, shoe modification, physical therapy and stretching exercises; completion of the ESWT protocol; grade of poor or fair in the Roles-Maudsley score (RMS) before the treatment (Table 1); and availability for long-term (at least 7 months after ESWT) follow-up telephone interviews. Exclusion criteria were history of previous steroid injections, tarsal tunnel syndrome, polyarthritis and rheumatic disease, previous surgery of the foot, lumbar spine disc herniation, trauma and pregnancy. The enrolled patients were divided into two groups according to US findings. Patients who met one of the Korean US diagnostic criteria for PF [20] were classified in the ApparentUS group and those who did not were classified in the Uncertain-US group (Fig. 1). Korean US diagnostic criteria for PF are plantar fascia thickness $>3.8 \mathrm{~mm}$ (Fig. 2), difference of plantar fascia thickness between the symptomatic and asymptomatic foot $>1.0 \mathrm{~mm}$ and hypoechogenicity in plantar fascia (Fig. 3).

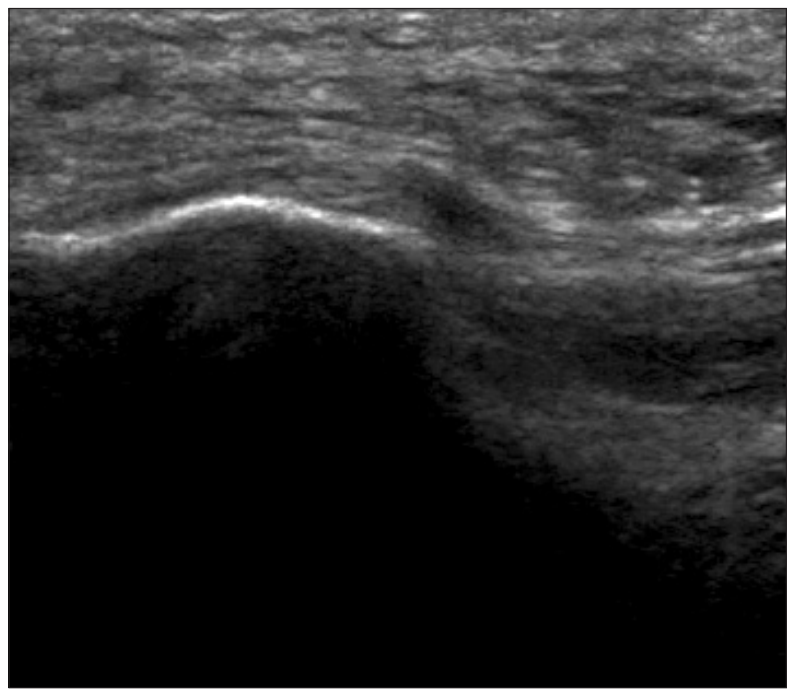

Fig. 1. Uncertain ultrasonographic finding that does not meet any of the Korean ultrasonographic diagnostic criteria for plantar fasciitis as detailed in the text. 


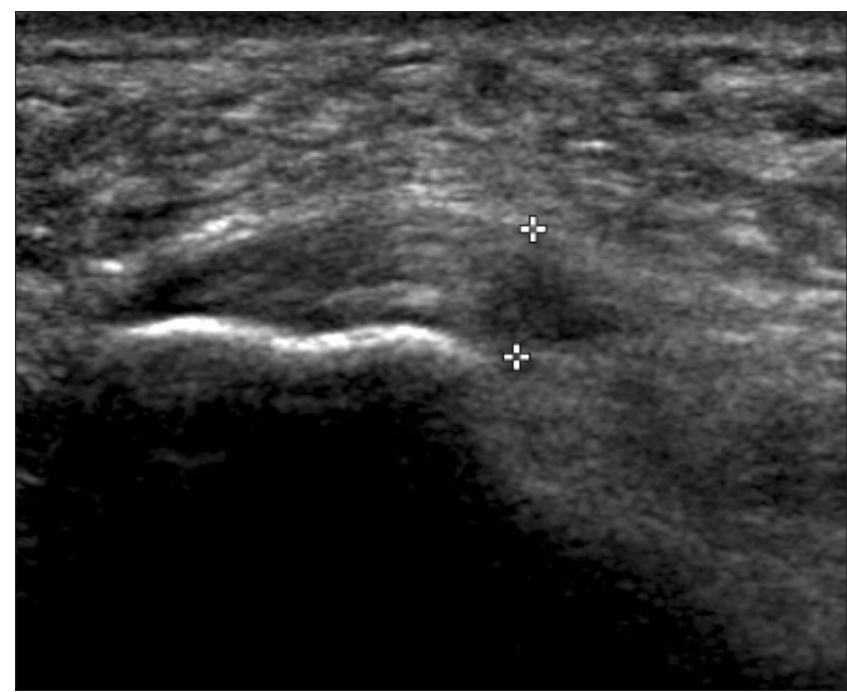

Fig. 2. Apparent ultrasonographic abnormal finding shows a plantar fascia thickness $>3.8 \mathrm{~mm}$.

\section{Sonographic evaluation}

Two musculoskeletal radiologists evaluated the plantar fascia of patients using a HDI 5000 (Philips Medical Systems, Bothell, WA, USA) and LOGIQ E9 (GE Healthcare, Milwaukee, WI, USA) imaging devices equipped with a variable frequency linear 6 to $15 \mathrm{MHz}$ probes. Patients were in prone position during examination with their feet hanging free over the end of the bed and their ankles dorsiflexed to $90^{\circ}$. Both sides were evaluated and compared with each other. Plantar fascia thickness was measured at its thickest point and echogenicity was assessed around the medial tubercle of the calcaneus.

\section{ESWT protocol}

No local anesthesia was applied. ESWT was performed using an Evotron electrohydraulic type apparatus (SwiTech Medical AG, Kreuzlingen, Switzerland) by one musculoskeletal physiatrist. Patients reclined in a prone position and their ankles were stabilized. The probe was chosen between EvoTrode R05 (penetration depth, 5-30 $\mathrm{mm}$ ) and R20 (penetration depth, 25-45 $\mathrm{mm}$ ) according to the depth of plantar fascia on US. The point of maximal heel tenderness was palpated and marked with a skin marker. US jelly was applied to the heel and low-energy ESWT $\left(0.10 \mathrm{~mJ} / \mathrm{mm}^{2}, 600\right.$ shocks $)$ was given once a week for 6 weeks. The frequency of shock was $1 \mathrm{~Hz}$. We tried to distribute the shock waves evenly over the whole of the tender area by adjustment of the probe.

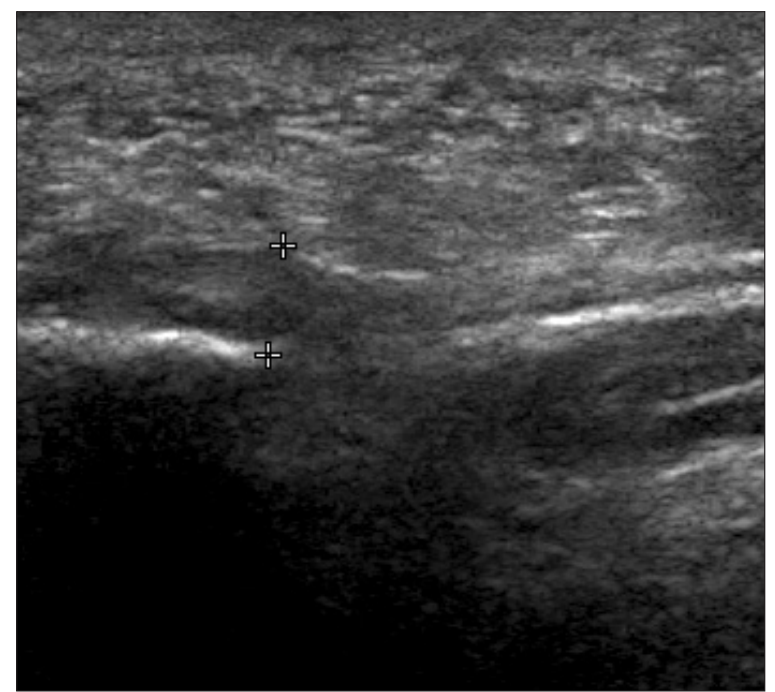

Fig. 3. Apparent ultrasonographic abnormal finding shows hypoechogenicity in the plantar fascia.

\section{Clinical assessment}

Pain intensity was measured before each ESWT session, and at short-term follow-up and long-term followup (telephone interview) using an 11-point pain intensity numerical rating scale (NRS), where 0 is no pain and 10 is worst possible pain. Short-term follow-up was conducted one week after completion of all ESWT sessions. Subsequently, long-term follow-up telephone interview was performed a mean of 24 months after ESWT (range, 7-42 months). RMS was also assessed before the first treatment, and at short-term and long-term follow-ups (Table 1). Overall success rate and success rate of each study group were calculated at short-term and long-term follow-ups. We defined a RMS grade of good or excellent as treatment success.

\section{Statistical analyses}

Independent t-test and repeated measure ANOVA test to compare and analyze the effect of low-energy ESWT between apparent-US and uncertain-US groups. The relationship among plantar fascia thickness, age, symptom duration and pain scores (NRS) were evaluated using Pearson correlation coefficient. All analyses were performed with the SPSS ver. 18.0 (SPSS Inc., Chicago, IL, USA). A p-value $<0.05$ was considered statistically significant. 


\section{RESULTS}

Table 2 shows baseline characteristics. All variables were not significantly different between Apparent-US and Uncertain-US group, except plantar fascia thickness. The results of repeated measure ANOVA test demonstrated that the subjective pain was significantly decreased with time up to long-term follow-up (mean 24 months after ESWT) (time effect, $\mathrm{p}<0.001$ ). However, there was no significant group-time interaction ( $\mathrm{p}=0.641$ for interaction), which means ESWT equally decreased subjective pain in both groups (Fig. 4). The pain score at short-term followup (one week after completion of all ESWT sessions) and long-term follow-up did not show significant difference between the two groups. These findings also indicated that there was no significant difference in the effect of lower-energy ESWT between two groups. In our study, overall success rate of ESWT was $63.3 \%$ at the short-term follow-up and $\mathbf{8 0 . 0 \%}$ at the long-term follow-up. In intergroup comparison, success rates at short-term follow-up were similar between Apparent-US (61.9\%) and Uncertain-US (66.7\%). At the long-term follow-up, those were $85.7 \%$ and $66.7 \%$, respectively (Table 3 ). There was no significant correlation between plantar fascia thickness on US, age, pain scores at each assessment and symptom duration.

\section{DISCUSSION}

Subjective pain started to decrease from 1 week after first treatment session and continued to improve with time up to mean 24 months. Using our definition of success, overall success rate at short-term follow-up (one week after completion of all ESWT sessions) and longterm follow-up (mean 24 months after ESWT) was $63.3 \%$ and $80.0 \%$, respectively. These results are comparable to previous reports that most patients improve within 2 weeks [21] and symptoms continued to improve with time to 6 months [22]. These findings are also consistent with other randomized control trials $[10,11,23,24]$ and a previous study that reported long-term symptom relief after ESWT [25].

US is often used for the diagnosis of PF by direct measurement of the thickness and observing echogenicity of the plantar fascia. Several studies [16-18,26-29] have consistently demonstrated a marked increase in plantar

Table 2. Basic characteristics according to ultrasonographic findings

\begin{tabular}{|c|c|c|c|c|}
\hline Variable & Apparent-US (n=21) & Uncertain-US (n=9) & Total $(n=30)$ & p-value e) $^{\text {a) }}$ \\
\hline Sex (male:female) & $3: 18$ & $4: 5$ & $7: 23$ & \\
\hline Lesion side (right:left) & $8: 13$ & 4:5 & $12: 18$ & \\
\hline Age (yr) & & & & 0.220 \\
\hline Mean (SD) & $52.7(9.8)$ & $47.3(12.6)$ & $51.1(10.8)$ & \\
\hline Range & $25-72$ & $21-61$ & $21-72$ & \\
\hline Symptom duration (mo) & & & & 0.281 \\
\hline Mean (SD) & $19.8(13.8)$ & $13.9(12.7)$ & $18.0(13.6)$ & \\
\hline Range & $6-36$ & $6-48$ & $6-48$ & \\
\hline Plantar fascia thickness (mm) & & & & $<0.001$ \\
\hline Mean (SD) & $4.4(0.8)$ & $3.0(0.6)$ & $4.0(0.1)$ & \\
\hline Range & $3.5-6.1$ & $2.0-3.7$ & $2.0-6.1$ & \\
\hline Initial NRS & & & & 0.614 \\
\hline Mean (SD) & $6.1(2.3)$ & $6.6(2.4)$ & $6.3(2.3)$ & \\
\hline Initial RMS (poor:fair) & $16: 5$ & $6: 3$ & $22: 8$ & \\
\hline Follow-up (mo) & & & & 0.500 \\
\hline Mean (SD) & $24.5(10.8)$ & $21.4(12.6)$ & $23.6(11.2)$ & \\
\hline Range & $7-41$ & $7-42$ & $7-42$ & \\
\hline
\end{tabular}

Apparent-US, apparent ultrasonographic abnormal group; Uncertain-US, uncertain ultrasonographic group; SD, standard deviation; NRS, numeric rating scale; RMS, Roles-Maudsley score.

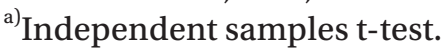


fascia thickness in symptomatic feet compared to asymptomatic sides. As a general rule, increased thickness ( $>4 \mathrm{~mm}$ ) and decreased echogenicity of plantar fascia are considered as consistent US findings in PF [28,29]. In Korean, Yoon et al. [20] reported that increased thickness $(>3.8 \mathrm{~mm})$, difference of thickness between symptomatic and asymptomatic sides $(>1.0 \mathrm{~mm})$ and hypoechogenicity of plantar fascia were clinically meaningful US findings

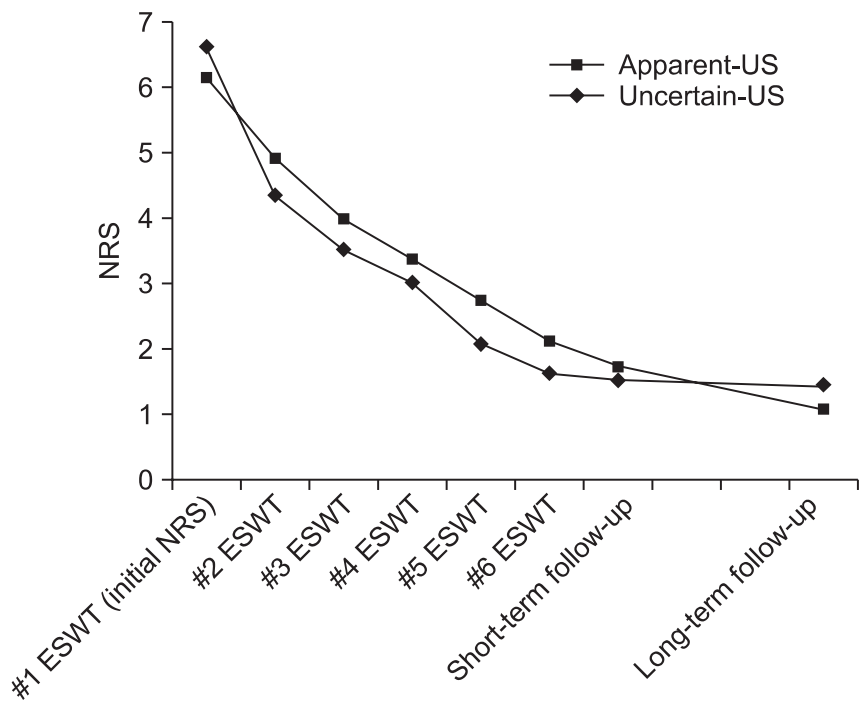

Fig. 4. Repeated measure ANOVA test demonstrates that pain score decreases with time up to long-term followup (mean 24 months after ESWT) (time effect, $\mathrm{p}<0.001$ ) without significant group-time interaction $(p=0.641$ for interaction). The pain scores at short-term follow-up (one week after completion of all ESWT sessions) and long-term follow-up do not show significant difference between the study groups. ESWT, extracorporeal shock wave therapy; NRS, numeric rating scale; Apparent-US, apparent ultrasonographic abnormal group; UncertainUS, uncertain ultrasonographic group. of PF. In the present study, we used these Korean criteria as the reference value for the determination of ApparentUS and Uncertain-US.

Repeated measure ANOVA revealed no significant group-time interaction ( $\mathrm{p}=0.641$ for interaction). In addition, the pain scores at short-term and long-term followups did not show a significant difference between the study groups. These findings suggest that ESWT equally improve subjective pain in both study groups regardless of presence or absence of apparent abnormalities on US, which are consistent with prior finding that plantar fascia thickness does not influence the outcome of ESWT [25]. However, the present results are contrary to the common understanding that plantar fascia thickness influences treatment outcomes [14]. Plantar fascia thickness can be marked increased in PF $[17,18]$ and substantially reduced after ESWT, showing a correlation with pain reduction $[30,31]$.

Several considerations about the main problem with US may to some extent explain such conflicting results. Operator-dependent technique and factors, such as lack of standardization of measurement points or machine settings, may affect the measured thickness value and appearance of plantar fascia, and make it difficult to determine reference values for designation of ApparentUS or Uncertain-US [32]. Indeed, large differences in the mean plantar fascia thickness were measured in the previous studies, in which the mean values varied from 2.9-8.1 mm in patients with PF. Ranges of plantar fascia thickness in patients with PF were $3.2-6.8 \mathrm{~mm}$ in one study [26] and 4.3-8.1 $\mathrm{mm}$ in another [27]. Yet another study reported a mean plantar fascia thickness of $2.9 \mathrm{~mm}$ in patients with unilateral heel pain [17]. Additionally, US evaluation of hypoechoic changes in the plantar fascia

Table 3. Success rate at short-term and long-term follow-ups according to ultrasonographic findings

\begin{tabular}{lccc}
\hline \multicolumn{1}{c}{ Follow-up } & Apparent-US (n=21) & Uncertain-US (n=9) & Total (n=30) \\
\hline Short-term & & & \\
Success rate (\%) & 61.9 & 66.7 & 63.3 \\
\hline Excellent & 1 & 0 & 1 \\
\hline Good & 12 & 6 & 18 \\
Long-term & & & 80.0 \\
Success rate (\%) & 85.7 & 66.7 & 4 \\
Excellent & 3 & 1 & 20 \\
\hline Good & 15 & 5 & 2 \\
\hline
\end{tabular}

Apparent-US, apparent ultrasonographic abnormal group; Uncertain-US, uncertain ultrasonographic group. 
was reported to display surprisingly low agreement [33], which could be attributed to the fact that the definition of echogenicity is dependent on the adjacent muscle, but there is no adjacent muscle for comparison when examining the plantar fascia with US. For these limitations, variations may exist among US examinations of the plantar fascia. Thus, proper and careful interpretation of findings is necessary when using US in routine clinical practice. Nevertheless, US is important to rule out other causes of heel pain or to establish the diagnosis of PF.

One limitation of the present study is the small number of patients studied. In addition, it has been reported that body mass index [34], walking hours per day, diabetes mellitus, and documented psychological disorder affect treatment outcome [25], but we did not consider these factors since this information was not available.

In conclusion, if other causes of heel pain are ruled out and diagnosis of PF is established through meticulous physical examination and US, low-energy ESWT appears to be beneficial regardless of US finding. In terms of success rate, however, long-term outcome of patients with apparent US abnormalities in plantar fascia appears to be superior to those with uncertain US findings.

\section{CONFLICT OF INTEREST}

No potential conflict of interest relevant to this article was reported.

\section{ACKNOWLEDGMENTS}

The authors thank Ms. Miyeon Lee, Medical Information Library in Kangbuk Samsung Hospital for her advice about statistical analyses used in the study.

\section{REFERENCES}

1. League AC. Current concepts review: plantar fasciitis. Foot Ankle Int 2008;29:358-66.

2. Pfeffer G, Bacchetti P, Deland J, Lewis A, Anderson $\mathrm{R}$, Davis W, et al. Comparison of custom and prefabricated orthoses in the initial treatment of proximal plantar fasciitis. Foot Ankle Int 1999;20:214-21.

3. Sorrentino F, Iovane A, Vetro A, Vaccari A, Mantia R, Midiri M. Role of high-resolution ultrasound in guiding treatment of idiopathic plantar fasciitis with mini- mally invasive techniques. Radiol Med 2008;113:48695.

4. Tsai WC, Wang CL, Tang FT, Hsu TC, Hsu KH, Wong MK. Treatment of proximal plantar fasciitis with ultrasound-guided steroid injection. Arch Phys Med Rehabil 2000;81:1416-21.

5. Radford JA, Landorf KB, Buchbinder R, Cook C. Effectiveness of calf muscle stretching for the short-term treatment of plantar heel pain: a randomised trial. BMC Musculoskelet Disord 2007;8:36.

6. Sperling JW, Antuna SA, Sanchez-Sotelo J, Schleck C, Cofield RH. Shoulder arthroplasty for arthritis after instability surgery. J Bone Joint Surg Am 2002;84:177581.

7. Babcock MS, Foster L, Pasquina P, Jabbari B. Treatment of pain attributed to plantar fasciitis with botulinum toxin a: a short-term, randomized, placebo-controlled, double-blind study. Am J Phys Med Rehabil 2005;84:649-54.

8. May TJ, Judy TA, Conti M, Cowan JE. Current treatment of plantar fasciitis. Curr Sports Med Rep 2002;1: 278-84.

9. Malay DS, Pressman MM, Assili A, Kline JT, York S, Buren B, et al. Extracorporeal shockwave therapy versus placebo for the treatment of chronic proximal plantar fasciitis: results of a randomized, placebocontrolled, double-blinded, multicenter intervention trial. J Foot Ankle Surg 2006;45:196-210.

10. Theodore GH, Buch M, Amendola A, Bachmann C, Fleming LL, Zingas C. Extracorporeal shock wave therapy for the treatment of plantar fasciitis. Foot Ankle Int 2004;25:290-7.

11. Rompe JD, Decking J, Schoellner C, Nafe B. Shock wave application for chronic plantar fasciitis in running athletes: a prospective, randomized, placebocontrolled trial. Am J Sports Med 2003;31:268-75.

12. Ogden JA, Alvarez RG, Levitt RL, Johnson JE, Marlow ME. Electrohydraulic high-energy shock-wave treatment for chronic plantar fasciitis. J Bone Joint Surg Am 2004;86:2216-28.

13. Haake M, Buch M, Schoellner C, Goebel F, Vogel M, Mueller I, et al. Extracorporeal shock wave therapy for plantar fasciitis: randomised controlled multicentre trial. BMJ 2003;327:75.

14. Buchbinder R. Clinical practice: plantar fasciitis. N Engl J Med 2004;350:2159-66. 
15. Hyer CF, Vancourt R, Block A. Evaluation of ultrasound-guided extracorporeal shock wave therapy (ESWT) in the treatment of chronic plantar fasciitis. J Foot Ankle Surg 2005;44:137-43.

16. Kamel M, Kotob H. High frequency ultrasonographic findings in plantar fasciitis and assessment of local steroid injection. J Rheumatol 2000;27:2139-41.

17. Ozdemir H, Yilmaz E, Murat A, Karakurt L, Poyraz AK, Ogur E. Sonographic evaluation of plantar fasciitis and relation to body mass index. Eur J Radiol 2005;54: 443-7.

18. Sabir N, Demirlenk S, Yagci B, Karabulut N, Cubukcu S. Clinical utility of sonography in diagnosing plantar fasciitis. J Ultrasound Med 2005;24:1041-8.

19. Karabay N, Toros T, Hurel C. Ultrasonographic evaluation in plantar fasciitis. J Foot Ankle Surg 2007;46:4426.

20. Yoon K, Kim SB, Park JS. Ultrasonographic findings in plantar fasciitis. J Korean Acad Rehabil Med 2002;26: 181-6.

21. Wang CJ, Wang FS, Yang KD, Weng LH, Ko JY. Longterm results of extracorporeal shockwave treatment for plantar fasciitis. Am J Sports Med 2006;34:592-6.

22. Chen HS, Chen LM, Huang TW. Treatment of painful heel syndrome with shock waves. Clin Orthop Relat Res 2001;(387):41-6.

23. Hammer DS, Adam F, Kreutz A, Kohn D, Seil R. Extracorporeal shock wave therapy (ESWT) in patients with chronic proximal plantar fasciitis: a 2-year follow-up. Foot Ankle Int 2003;24:823-8.

24. Cosentino R, Falsetti P, Manca S, De Stefano R, Frati E, Frediani B, et al. Efficacy of extracorporeal shock wave treatment in calcaneal enthesophytosis. Ann Rheum Dis 2001;60:1064-7.

25. Chuckpaiwong B, Berkson EM, Theodore GH. Extra- corporeal shock wave for chronic proximal plantar fasciitis: 225 patients with results and outcome predictors. J Foot Ankle Surg 2009;48:148-55.

26. Cardinal E, Chhem RK, Beauregard CG, Aubin B, Pelletier M. Plantar fasciitis: sonographic evaluation. Radiology 1996;201:257-9.

27. Gibbon WW, Long G. Ultrasound of the plantar aponeurosis (fascia). Skeletal Radiol 1999;28:21-6.

28. Tsai WC, Chiu MF, Wang CL, Tang FT, Wong MK. Ultrasound evaluation of plantar fasciitis. Scand J Rheumatol 2000;29:255-9.

29. Akfirat M, Sen C, Gunes T. Ultrasonographic appearance of the plantar fasciitis. Clin Imaging 2003;27:3537.

30. Hammer DS, Adam F, Kreutz A, Rupp S, Kohn D, Seil R. Ultrasonographic evaluation at 6-month followup of plantar fasciitis after extracorporeal shock wave therapy. Arch Orthop Trauma Surg 2005;125:6-9.

31. Liang HW, Wang TG, Chen WS, Hou SM. Thinner plantar fascia predicts decreased pain after extracorporeal shock wave therapy. Clin Orthop Relat Res 2007;460:219-25.

32. Scheel AK, Schmidt WA, Hermann KG, Bruyn GA, D'Agostino MA, Grassi W, et al. Interobserver reliability of rheumatologists performing musculoskeletal ultrasonography: results from a EULAR "Train the trainers" course. Ann Rheum Dis 2005;64:1043-9.

33. Cheng JW, Tsai WC, Yu TY, Huang KY. Reproducibility of sonographic measurement of thickness and echogenicity of the plantar fascia. J Clin Ultrasound 2012; 40:14-9.

34. Riddle DL, Pulisic M, Pidcoe P, Johnson RE. Risk factors for plantar fasciitis: a matched case-control study. J Bone Joint Surg Am 2003;85:872-7. 